\title{
MULTISPECTRAL ANALYSIS OF INDIGENOUS ROCK ART USING TERRESTRIAL LASER SCANNING
}

\author{
B. Skoog, P. Helmholz*, D. Belton \\ Department of Spatial Sciences, Curtin University, GPO Box U 1987, Perth WA 6845, Australia \\ (Bjorn.Skoog, Petra.Helmholz, D.Belton)@ curtin.edu.au
}

Commission V, WG V/2

KEY WORDS: Terrestrial Laser Scanning, Data Fusion, Classification, Heritage Mapping

\begin{abstract}
:
Multispectral analysis is a widely used technique in the photogrammetric and remote sensing industry. The use of Terrestrial Laser Scanning (TLS) in combination with imagery is becoming increasingly common, with its applications spreading to a wider range of fields. Both systems benefit from being a non-contact technique that can be used to accurately capture data regarding the target surface. Although multispectral analysis is actively performed within the spatial sciences field, its extent of application within an archaeological context has been limited. This study effectively aims to apply the multispectral techniques commonly used, to a remote Indigenous site that contains an extensive gallery of aging rock art. The ultimate goal for this research is the development of a systematic procedure that could be applied to numerous similar sites for the purpose of heritage preservation and research. The study consisted of extensive data capture of the rock art gallery using two different TLS systems and a digital SLR camera. The data was combined into a common 2D reference frame that allowed for standard image processing to be applied. An unsupervised k-means classifier was applied to the multiband images to detect the different types of rock art present. The result was unsatisfactory as the subsequent classification accuracy was relatively low. The procedure and technique does however show potential and further testing with different classification algorithms could possibly improve the result significantly.
\end{abstract}

\section{INTRODUCTION}

Terrestrial Laser Scanning (TLS) is a widely used tool in today's surveying industry. Increasing usage of TLS has instigated extensive research with Light Detection And Ranging (LiDAR) technology, resulting in applications ranging from building modelling, deformation monitoring, terrain mapping and heritage preservation (Armesto-González, 2010). Archaeological usage specifically has increased significantly in the past decade, with numerous heritage sites being captured worldwide (Briese, 2013). To date though, the majority of archaeological TLS use has been limited to recording basic 3D geometric information for modelling, visual interpretation and preservation.

While TLS data provides valuable 3D point information, it also includes the returning signal intensity information. This intensity data has the potential to be used for determining additional information regarding the target surface (Burton, 2011). At current the use of intensity information is predominantly restricted to research level evaluation; however its application to heritage sites could prove invaluable.

While LiDAR intensity information is used widely in airborne and remote sensing applications, the need for in-depth research into the calibration and analysis of the data is becoming considerably more prominent. At current archaeological site recording is limited to images and $3 \mathrm{D}$ scans. The goal of this paper is to expand on current methods, by capturing a virtual model of the rock art, and also use it to be able to detect rock art which has undergone significant levels of superimposition and fading due to weathering. If successful, virtual models that display and improve existing site knowledge could be created and kept for tourism and future generations to view.

The outline of this paper is as follow: After related work is discussed in section 2, the test site and the captured data is presented in section 3. Section 4 introduced the methodology which is evaluated in section 5. A conclusion is given in section 6.

\section{RELATED WORK}

For the majority of applications, the primary use for TLS has been to provide basic 3D point geometry information of the target region being acquired (Vosselman, 2010). The use of the intensity return associated with each $3 \mathrm{D}$ point has been relatively limited, with most projects being restricted to singular intensity wavelength analysis. As the intensity return can be affected by the target surface reflectance values, the incidence angle, distance to target and atmospheric conditions (Kaasalainen, 2010), analysis of such data quickly becomes a complex multi-faceted problem to solve. Instrument calibration factors in heavily, especially if meaningful and accurate results are to be produced (Höfle, 2007).

According to (Armesto-González, 2010), the effectiveness of intensity analysis does not appear to be restricted to certain wavelengths. Three different TLS systems were utilising for the damage detection of historical buildings. Generic unsupervised classification algorithms were applied to the 2D intensity images extracted from the individual 3D point cloud datasets. Different segments from within the historical building were able to be easily recognised and highlighted for further analysis.

\footnotetext{
* Corresponding author
} 
Important to note is that none of the three datasets were calibrated, yet still generated acceptable classification results.

Testing of spectral analysis within a geological application has been performed extensively in a research capacity due to its potential usefulness for lithographic examination. The majority of testing has been limited to single spectrum analysis. A single laser scanner for spectral analysis was used by Franceschi et al., (2009). The geological target surface was a rock outcrop that contained alternating layers of marls and limestone. The ground truth was established using gas chromatography testing of the two different clay types. The differing intensity values were then used to distinguish between alternating layers by generating a reflectance series along the rock outcrop. The intensity values were distance corrected. The two different components were then segmented with a clear correlation. Importantly the target surface and environment were thoroughly considered in the trial with target distance, incidence angle, Lambertian surface behaviour, humidity and temperature. Thermal imagery was captured throughout the testing to check for a temperature correlation, but was proven as unrelated. Target moisture did factor in considerably as the scanner operates within the infrared wavelength, meaning moisture on the target acts to absorb part of the emitted laser.

The independence of the incidence angle to the target surface, when the target surface displayed Lambertian surface behaviour, was also confirmed by Hartzell et al. (2014). This paper performed multispectral testing using three different laser scanners, along with a digital SLR camera to produce 2D 6band images. The test area was a rock outcrop composed of three distinct geological materials. The point cloud datasets were also radio-metrically calibrated using a spectroradiometer. Two different unsupervised classification algorithms were implemented, one was the Minimum Distance algorithm and the other was Maximum Likelihood, and both highlighted the 3 different lithographic sections clearly. When the incidence angle was incorporated into the calibration component, it resulted in a decrease of the final classification accuracy.

González-Jorge et al. (2012) performed an intensity analysis but applied it to biological crusts located on civil engineering structures. Two different TLS systems were utilised to capture areas that had significant amounts of biological crust developing. The 3D scans were converted into $2 \mathrm{D}$ intensity images and two different unsupervised classification algorithms were tested in order to detect the biological crusts on the structure. Results varied depending on the scanner used as one operated within the visible green wavelength and the second was within the infrared wavelength. This would cause a significantly different result as the infrared wavelength is heavily absorbed by the moisture present in the biological crusts. Hence, the trial highlights the different results that are caused by use of different wavelength scanners.

Kaasalainen et al. (2010) performed a detailed study of the effects of scanning targets with varying percentage levels of moisture saturation to determine the effect on the returning intensity values. It was found that when using a near-infrared TLS there was a significant decrease in the backscattered reflectance values, but it depended strongly on both the target material composition and the level of moisture content. The returning intensity was reduced by more than $50 \%$ in some cases, with the effect of moisture saturation having minimal impact beyond a level of $10-12 \%$. This indicates that if scanning was performed over a moist target surface it has to be calibrated, especially if the TLS was operating within the nearinfrared or infrared wavelength range.

An issue that multiple studies have consistently raised (Kukko, 2008; Soudarissanane, 2011; Teza, 2008; Kaasalainen, 2011) is the effect of incidence angle on the returning laser scanner intensity. Kukko et al. (2008) determined quantitatively the significance of the incidence angle by scanning calibrated reflectance targets with different laser systems at varying incidence angles. For incidence angles of up to $30^{\circ}$ the effect was minimal, but beyond that the returning intensity decreased significantly. This indicates that any scanning performed at any significant incidence angle would need to have its intensity values corrected based on the surface normals at the target surface. (Soudarissanane, 2011) undertook similar testing of incidence angles and combined distance effects as well. The incidence angle testing displayed similar results. The distance $(R)$ testing showed significant variations in the intensity return (I) depending of the distance from the scanner to the target. For distances below $10 \mathrm{~m}$, the intensity values do not follow the expected trend of $I / R^{2}$. However, beyond $10 \mathrm{~m}$ the intensity values approximate towards the expected trend. From this it can be established that distance related calibration is essential for obtaining meaningful results from intensity data, where situations that exhibit extreme incidence angles and varying distances over the target field will cause inconsistences in the intensity values.

When performing classification of various data, the list of available algorithms is extensive and varied depending on their application. Lu et al. (2007) presents a survey of existing algorithms and their applications. The classification of 2D intensity imagery has been done extensively in the photogrammetric and remote sensing fields, typically with airborne imagery and LiDAR. Gerke et al. (2014) presents a LiDAR classification case study utilising supervised classification techniques. Although this study used the combined terrain height information and intensity classification for the final result, the intensity processing can be considered for relevance. Nearly all of the different terrain areas were correctly classified with minimal misclassification.

Gerke et al. (2014) performs a detailed analysis of the initial point cloud filtering procedure and the resultant classification process and result. 4-band spectral data was used from the TLS, being RGB from the internal camera and infrared from the scanner itself. From the original data, a training dataset was generated and used for the supervised classification (maximum likelihood). For classes that demonstrated similar spectral signatures, misclassification occurred frequently with an overall accuracy of only $60 \%$ being correct. When analogous classes were merged, the accuracy improved significantly to $82 \%$. This demonstrates that when using a minimal number of relatively distinct classes fairly accurate data classification can be applied of multispectral data.

A comparison of multispectral versus multiband supervised classification was performed by (Lerma, 2001) using typically architectural imagery. Although this testing has been applied in a photogrammetric context, the process applies itself to TLS data the same way. The training data were generated from several unique feature sets identified with the images and used in a supervised maximum likelihood classification. The resultant classification accuracies were similar for both the multispectral and multiband, with the multiband images producing slightly improved classification. However, this is 
purely an empirical result and only applies if the multiband imagery used was captured under suitable conditions.

In this paper we utilise multispectral analysis of TLS intensity data captured from Indigenous rock by two different scanners, along with digital images of the same target area. The scanners utilised operate on a different wavelength offering intensity differences. The aim is by utilising this difference, to extract and plot features in the rock art generating a digital record for additional interpretation and site preservation. This is especially important in Australia as many protected Indigenous sites are suffering due to high tourist loads or are located in areas highly sought after by mining companies due to nearby mineral deposits.

\section{TEST SITE AND DATA}

The test site is Walga Rock located approximately $700 \mathrm{~km}$ to the north east of Perth in Western Australia. On the NW face of the rock is an approximately $100 \mathrm{~m}$ long gallery of Rock Art of different ages and different colours. The Rock Art site is protected and only non-contact methods are allowed for data capturing.

The first component required establishing a suitable and stable control network at the Walga Rock site that could be utilised to register the TLS and imagery data together and geo-reference it. The control was established using traditional survey methods. A total of 20 control points were surveyed with a Total Station (TS), with 12 points located directly in front of the gallery and the remaining eight points located further away from the site. Two of the stations were used to perform static GNSS baseline observations to a nearby known standard survey mark (SSM) in order to provide georeferenced coordinates. Additionally, differential levelling was performed between the points in order to transfer heights accurately for the survey.

Processing of the survey data consisted of static GNSS baseline processing in Trimble Business Centre (TBC) in order to transfer coordinates from the nearby SSM. The full TS and levelling dataset was then inputted into an advanced least squares adjustment software called Geolab PX5 for processing. This resulted in full coordinates for all the stations in the control network that could then be used for registering the TLS and image data. The network had a large redundancy of 71 due to the extensive number of observations that were taken in the field. The adjustment resulted in station precisions with a maximum of $0.004 \mathrm{~m}$ for the horizontal semi-major axis error ellipses, and a maximum of $0.003 \mathrm{~m}$ for the vertical error bars. These values are stated at the $95 \%$ confidence interval, with the network containing no outlying observations as per Baarda's data snooping of the standardised residuals. The network also passed the global variance factor test. In terms of survey precision as stated by the Standards and Practices for Control Surveys (SP1) (ICSM, 2007), the network meets class A horizontally and class LC vertically.

The laser scans were captured using the Leica C10 (green wavelength) and the Trimble TX5 (infrared wavelength) TLS systems. This consisted of performing high density laser scans of the rock art at each of the 12 stations, using both of the scanners. This resulted in a total of 58GB of raw scan data for the entire rock art gallery. The raw scan data from each TLS were registered separately into a common local reference frame using point cloud processing software Cyclone. The resulting registrations were joined in a single registration and georeferenced using the adjusted control coordinates. From this complete georeferenced point cloud, different segments could be extracted where desired for further processing. The mean absolute error resulting from the final registration was $0.005 \mathrm{~m}$. Considering the size of the target area and number of scans involved, this is an acceptable error magnitude. The number of points for the Leica point cloud totalled $\sim 1,250,000,000$ and the Trimble point cloud had $\sim 6,000,000,000$ points. The difference in numbers is due to the scan density that each scanner could be set to and the data capturing method (time of flight vs. phase difference).

The images from the rock art gallery consist of over 300 captured using two separate digital SLR cameras. The images were captured with varying lighting, so that in the end only a limited number were actually used for the testing. These images were captured by a Nikon D80 camera. Standard photogrammetric procedures were followed during data capturing. For instance, a scale bar was placed in multiple images. The image processing was completed using the photogrammetric software iWitnessPro. This involved importing the images and performing relative orientation using common feature points. The georeferenced point clouds were used to pick common points on the Rock Art, which were used to provide absolute orientation in a bundle adjustment with selfcalibration. Post calibration accuracy achieved was an RMS of 0.27 pixels which indicates a satisfactory image alignment was obtained. This enabled the images to be easily combined with the point cloud dataset as both have been georeferenced to the same object coordinate system.

\section{METHODOLOGY}

\subsection{D to 2D transformation and data fusion}

Having referenced both (3D and 2D) datasets into a common system, the $3 \mathrm{D}$ dataset can be transformed into a $2 \mathrm{D}$ form in order for ease of processing. By performing a transformation into $2 \mathrm{D}$, it will enable the use of common photogrammetric and remote sensing image processing techniques to be applied.

Prior to transforming the 3D point cloud data into $2 \mathrm{D}$, the corresponding image scene was used to colour the point cloud. This was done within Cyclone's texture map toolbox. This allows for a simple projective transformation to be applied to the image and map the projective image onto the corresponding 3D point cloud. This was applied by using a minimum of ten corresponding tie points in both the image and the point cloud. The resultant point cloud contained data corresponding to intensity, as well as RGB values from the image scene.

As the imagery and point cloud data sets in the same object coordinate system, it can be easily transformed into $2 \mathrm{D}$ image space. To perform this transformation, the same image of the art rock was considered as the base with which the 3D data will be transformed onto. The parameters of the IO and EO were used to determine the transformation parameters that were applied using the collinearity equations to the high density scan that covered the same area. This transformed the entire scan into the same image coordinate system as the image. The principal point offset was applied along with the radial lens distortion parameters, as defined from the camera calibration done previously. The 2D point cloud was run through an extent reduction procedure. This consisted of eliminating data that lay beyond the extents of the original base image. To perform this, the data had its extents reduced to the dimensions as defined by 
the number of pixels defining the image size. This produced a scattered $2 \mathrm{D}$ raster image that covered the same extents as the base image.

In order to applied generic image feature processing, the images used for processing must have the same image dimensions. In order to convert to an organised raster image a nearest neighbour grid interpolator was applied. This resulted in a multiband 2D raster image, with each pixel being assigned an intensity value and RGB value based on the interpolation.

\subsection{Image enhancement}

Prior to further processing of the multiband 2D image scene, image enhancement was performed. This was done by inspecting the intensity histograms for each individual band and performing an adjustment in order to improve the overall scene contrast. Three different techniques of contrast enhancement were applied in order to improve the contrast of each individual band. The first method (imadjust) operates by mapping the intensity values to a new set of values where $1 \%$ of the original data is saturated at the low and high intensities of the original image. The second algorithm (histeq) operates by transforming the intensity values within an image, such that the resultant histogram of intensities is forced to approximately agree with a predefined histogram shape. The third technique (adapthisteq) works on the intensity values by transforming them using a contrast-limited adaptive histogram equalisation.

\subsection{Unsupervised classification}

The K-means unsupervised clustering algorithm was used for the classification of the images. The algorithm was applied to different combinations of three band images from the original five bands that were created. Different numbers of clusters were also implemented to determine the most effective result. Inspection of the image scene being tested revealed three to five different classes depending on interpretation and whether similar features were considered a single class or considered as distinct. The distance between the mean centroid clusters was determined by the default squared Euclidean distance metric.

\section{RESULTS}

\subsection{Ground truth}

Determination of ground truth was implemented for a small segment located within one of the image scenes due to the test site being large and thus generating ground truth for the entire area was not a practical solution given the task scope (and is indeed a challenge even for experts of this Rock Art). The test site selection was determined based on areas that had a large amount of artwork within the scene, contained areas of relatively high contrasting features within the image and also consisted of all the different classes at the target site.

For the ground truth, a small region was selected and the different classes in the area clearly segmented. The identified classes were exported and run through the same 3D to $2 \mathrm{D}$ transformation procedure describe earlier, in order to bring them to the same image coordinate system for comparison with the classification result.

\subsection{D to 2D transformation and data fusion}

Shown in Figure 1 is the result of the texture mapping of the images onto the $3 \mathrm{D}$ point cloud. Each point of the coloured point cloud has a RGB values associated along with the original intensity values from the scanners. The accuracy of the colour mapping was stated at 1.1 pixels. Although ideally a value below 1 pixel is sought, considering the projective distortions present in the image and any unseen artefacts in the point cloud, this is an acceptable accuracy level.

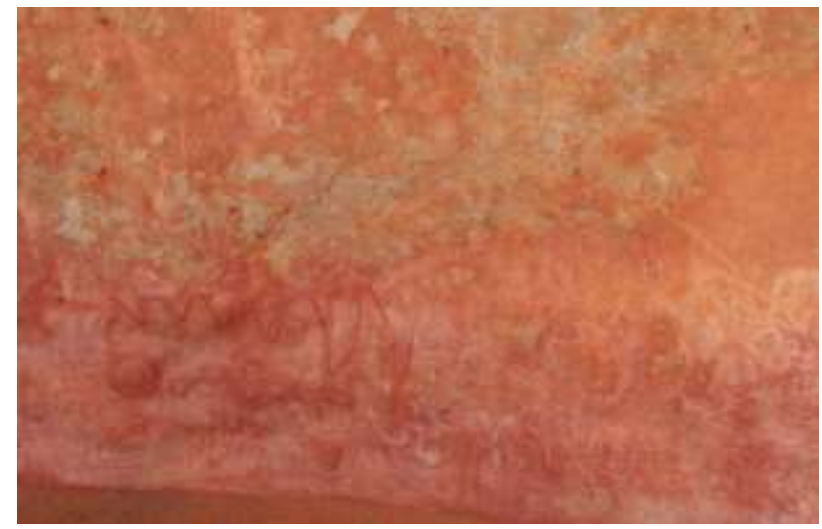

Figure 1. Screenshot of the coloured point cloud.

After colour mapping and subsequent exporting of the point cloud data, the collinearity equations were used to perform the $3 \mathrm{D}$ to $2 \mathrm{D}$ transformation. The resultant 5 image bands are used to perform the image classification next. Figure 2 shows the five channel images in the left column.

\subsection{Image enhancement}

Inspecting of the five different bands highlights different components of the scene. While the blue and green band display the white rock art with more contrast, the Leica band displays the darker rock art more clearly. The Trimble band does contain an artefact in the lower left of the image. This was caused by missing data from the point cloud, however as it does not cover the primary target surface, it will not impact the classification result. From each of the 5 bands the corresponding normalised intensity histograms are shown in Figure 2 in the right column.

By visual inspection, the histogram spreads fall into two distinct categories based on the instrument used to capture the particular band. For the red, green and blue bands a wider spread across the greyscale intensity is clearly visible. In contrast, for the two TLS based intensity profiles the shape is considerably narrower. This is due to the technical properties of the two capture methods. The RGB based bands were captured with a standard digital SLR camera. The wavelength range that each of these three bands covers is relatively large and may cover up to $100 \mathrm{~nm}$ of the light spectrum. The TLS however, provides its own precise wavelength when the laser is emitted.

Afterwards, three different histogram adjustment techniques (imadjust, histeq and adapthisteq) were used. Visual inspection led to the conclusion that the first two techniques only provide minimal enhancement as too much of the image detail is lost due to over saturated areas or regions that are too dark. The third technique displays a positive result with greatly enhanced contrast shown between the artwork and the surrounding area. The same algorithm was tested and successfully applied to the remaining red, green, Leica and Trimble image bands. 

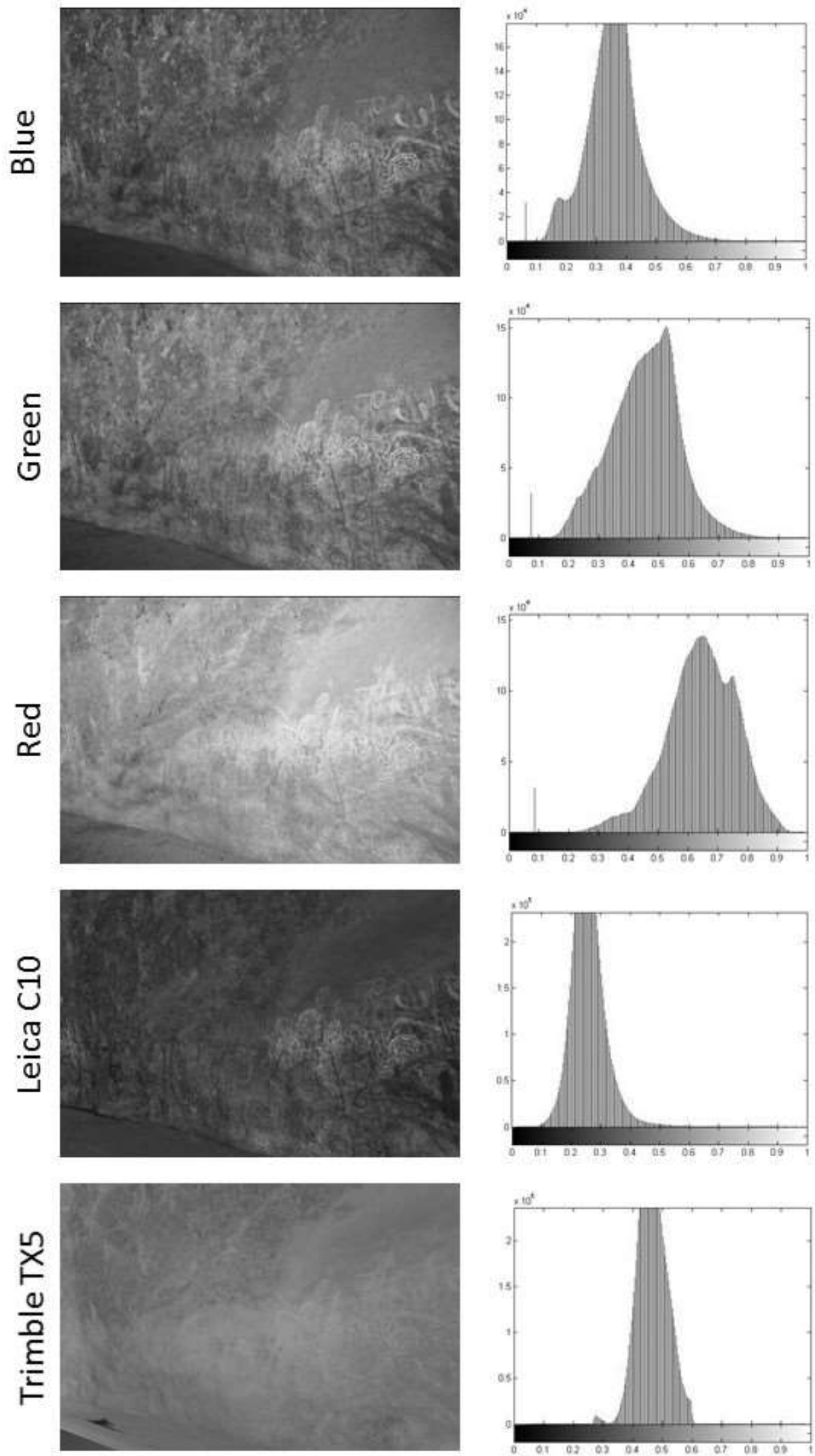

Figure 2: Images of the different bands with the corresponding normalised intensity histograms (histograms are normalised to range from 0 to 1 ). 


\subsection{Unsupervised classification}

Before the unsupervised classification was applied, different three-band-images as a combination of the above explained five band images were created. In addition the number of clusters for the k-mean algorithm was chosen. In total five tests were conducted using the combinations shown in Table 1.

\begin{tabular}{|c|l|c|}
\hline Test & Bands & \# clusters \\
\hline 1 & $\begin{array}{l}\text { Enhanced blue, enhanced Leica \& } \\
\text { enhanced Trimble }\end{array}$ & 3 \\
\hline 2 & Leica and Trimble and enhanced blue & 3 \\
\hline 3 & Leica and Trimble and enhanced blue & 4 \\
\hline 4 & Leica and enhanced blue and red & 4 \\
\hline 5 & Enhanced red, green and blue & 4 \\
\hline
\end{tabular}

Table 1: Bands configuration for the tests performed.

Figure 3 shows the classification result for test 1 . While the figure presents the whole scene, for the calculation of the confusion matrix in Table 2 only a small part of the scene was used in which the ground truth data was available.

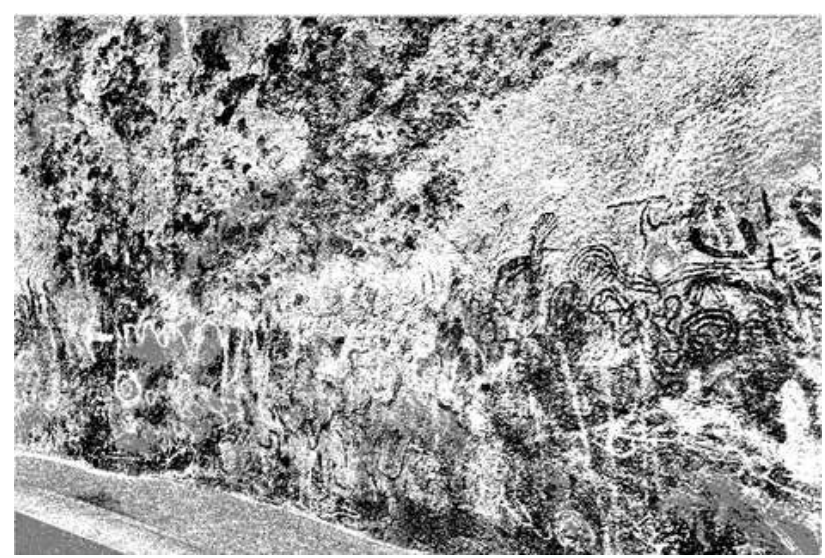

Figure 3: cluster result using enhanced blue, Leica and Trimble bands

Table 2 shows confusion matrix for test 1 that quantifies the effectiveness of the three clusters including their producer's and user's accuracy levels. The overall accuracy is $56.9 \%$ and the kappa coefficient is 0.35 . It can be confirmed that while the white artwork was extracted to a reasonably successful level, the remaining portions from the image scene were heavily misclassified and give a poor overall result. Overall the classification results not successful.

\begin{tabular}{|c|c|c|c|}
\hline & $\begin{array}{c}\text { White } \\
\text { artwork }\end{array}$ & $\begin{array}{c}\text { Chipped } \\
\text { rock }\end{array}$ & $\begin{array}{c}\text { Red } \\
\text { rock/artwork }\end{array}$ \\
\hline Cluster 1 & $80.9 \%$ & $50.7 \%$ & $14.9 \%$ \\
\hline Cluster 2 & $11.6 \%$ & $42.9 \%$ & $38.2 \%$ \\
\hline Cluster 3 & $7.6 \%$ & $6.4 \%$ & $46.9 \%$ \\
\hline \hline Producer's A. & $80.9 \%$ & $42.9 \%$ & $46.9 \%$ \\
\hline User's A. & $55.2 \%$ & $46.3 \%$ & $77.0 \%$ \\
\hline
\end{tabular}

Table 2: Confusion matrix test 1 .

Test 2 uses the same spectral bands whereas only the blue channel is enhanced, but not the Leica and Trimble channel and could achieve slightly better results as presented in Table 3 . In this case the overall accuracy is $61.0 \%$ and the kappa coefficient is 0.42 . Again white artwork was classified the best whereas the classification results of Chipped rock and Red rock/artwork also increases.

\begin{tabular}{|c|c|c|c|}
\hline & $\begin{array}{c}\text { Chipped } \\
\text { rock }\end{array}$ & $\begin{array}{c}\text { White } \\
\text { artwork }\end{array}$ & $\begin{array}{c}\text { Red } \\
\text { rock/artwork }\end{array}$ \\
\hline Cluster 1 & $52.4 \%$ & $22.2 \%$ & $35.2 \%$ \\
\hline Cluster 2 & $23.6 \%$ & $74.8 \%$ & $8.9 \%$ \\
\hline Cluster 3 & $24.0 \%$ & $3.0 \%$ & $55.9 \%$ \\
\hline Producer's A. & $52.4 \%$ & $74.8 \%$ & $55.9 \%$ \\
\hline User's A. & $47.7 \%$ & $69.7 \%$ & $67.5 \%$ \\
\hline
\end{tabular}

Table 3: Confusion matrix test 2.

As the results for test 2 increases the same band combination was used also for test 3 , but this time the number of classes was increased to four. The joint class Red rock/artwork was split into two separate classes. The results are presented in Table 4; the overall accuracy for this classification was $51.6 \%$ and the kappa coefficient was $0.36 \%$, i.e. the classification accuracy decreased. Especially the results for Red rock are unsatisfying.

\begin{tabular}{|c|c|c|c|c|}
\hline & $\begin{array}{c}\text { White } \\
\text { artwork }\end{array}$ & $\begin{array}{c}\text { Red } \\
\text { rock }\end{array}$ & $\begin{array}{c}\text { Red } \\
\text { artwork }\end{array}$ & $\begin{array}{c}\text { Chipped } \\
\text { rock }\end{array}$ \\
\hline Cluster 1 & $73.8 \%$ & $10.2 \%$ & $0.9 \%$ & $22.0 \%$ \\
\hline Cluster 2 & $1.8 \%$ & $22.3 \%$ & $31.0 \%$ & $22.9 \%$ \\
\hline Cluster 3 & $1.9 \%$ & $27.9 \%$ & $60.8 \%$ & $4.6 \%$ \\
\hline Cluster 4 & $22.6 \%$ & $39.7 \%$ & $7.4 \%$ & $50.5 \%$ \\
\hline Producer's A. & $73.8 \%$ & $22.3 \%$ & $60.8 \%$ & $50.5 \%$ \\
\hline User's A. & $69.1 \%$ & $28.6 \%$ & $63.9 \%$ & $42.0 \%$ \\
\hline
\end{tabular}

Table 4: Confusion matrix test 3.

A visual inspection of Figure 2 indicates that the combination of "Leica and enhanced blue and red" should lead to good results, which was evaluated in test 4 . The results are shown in Table 5. The overall accuracy is $54.9 \%$ and the kappa coefficient is 0.40 . This is a slightly better result as in the previous test indicating that the red band is more valuable than the infrared information from the Trinble TX5 band. The classification results of the Red rock improved while the results for Chipped rock decreased.

\begin{tabular}{|c|c|c|c|c|}
\hline & $\begin{array}{c}\text { White } \\
\text { artwork }\end{array}$ & $\begin{array}{c}\text { Red } \\
\text { rock }\end{array}$ & $\begin{array}{c}\text { Red } \\
\text { artwork }\end{array}$ & $\begin{array}{c}\text { Chipped } \\
\text { rock }\end{array}$ \\
\hline Cluster 1 & $74.7 \%$ & $1.7 \%$ & $19.2 \%$ & $29.1 \%$ \\
\hline Cluster 2 & $1.1 \%$ & $69.2 \%$ & $17.6 \%$ & $15.2 \%$ \\
\hline Cluster 3 & $16.3 \%$ & $29.2 \%$ & $63.2 \%$ & $43.2 \%$ \\
\hline Cluster 4 & $7.8 \%$ & $0.0 \%$ & $0.0 \%$ & $12.5 \%$ \\
\hline \hline Producer's A. & $74.7 \%$ & $69.2 \%$ & $63.2 \%$ & $12.5 \%$ \\
\hline User's A. & $59.9 \%$ & $67.1 \%$ & $41.6 \%$ & $61.6 \%$ \\
\hline
\end{tabular}

Table 5: Confusion matrix test 4 .

\begin{tabular}{|c|c|c|c|c|}
\hline & $\begin{array}{c}\text { White } \\
\text { artwork }\end{array}$ & $\begin{array}{c}\text { Red } \\
\text { artwork }\end{array}$ & Red rock & $\begin{array}{c}\text { Chipped } \\
\text { rock }\end{array}$ \\
\hline Cluster 1 & $95.7 \%$ & $24.2 \%$ & $2.8 \%$ & $96.0 \%$ \\
\hline Cluster 2 & $0.2 \%$ & $17.8 \%$ & $8.1 \%$ & $3.1 \%$ \\
\hline Cluster 3 & $4.1 \%$ & $51.0 \%$ & $58.8 \%$ & $0.9 \%$ \\
\hline Cluster 4 & $0.0 \%$ & $7.0 \%$ & $30.3 \%$ & $0.0 \%$ \\
\hline Producer's A. & $95.7 \%$ & $17.8 \%$ & $58.8 \%$ & $0.0 \%$ \\
\hline User's A. & $43.8 \%$ & $61.1 \%$ & $51.2 \%$ & $0.0 \%$ \\
\hline
\end{tabular}

Table 6: Confusion matrix test 5 .

The final test (test 5) shows the result for the classification without the use of any of the scanner bands (Table 6). The result is the worst with an overall accuracy of $43.1 \%$ and a 
kappa coefficient of 0.24 . There is a high confusion in Red artwork and also Red rock. Chipped rock and White artwork got completely mixed up visible in a producer's and user's accuracy of Chipped rock of $0.0 \%$.

\section{CONCLUSIONS}

An investigation into the effectiveness of utilising multispectral data for feature extraction has been completed. This was achieved through the use of two different laser scanners and a digital SLR camera to perform the data capture. The combined multiband dataset was run through a generic unsupervised classification algorithm (k-means) that extracted clusters from within the dataset for comparison to the ground truth. The resultant clustering was then cross-validated with a ground truth and a classification accuracy produced.

The end result of the classification accuracy was unsatisfactory for all combinations of image bands that were trialled. None yielded an overall accuracy of above $61 \%$, with the highest kappa coefficient being 0.42 . Individual classification accuracy was higher in certain cases, especially with the white rock art. However the significant level of misclassification by the remaining classes had the tendency to pollute the final result too much.

The method described indicates that there is significant potential for the feature extraction and classification of Indigenous rock art using a similar technique. However, the end result needs improvement. Firstly, further unsupervised algorithms should be trialled. Nevertheless, due to the nature of the data which contained significant amounts of superimposition and eroding, the improved effectiveness of a different unsupervised classifier may be limited. The use of a supervised classification algorithm may hold more promise. By using training data that takes into account the subtle variability within the dataset, the classification result could be significantly improved.

Secondly, while the large amount of TLS data captured was initially appealing, the high density proved to be excessive. It did cause issues when processing in the form of time delays and the extremely high redundancy proved unnecessary as the image data could not provide the same level of detail as the point cloud data.

However, the extensive 3D dataset generated has plenty of further potential use. The dataset can be used as an archaeological record for the site should any damage occurs. The dataset also has a very worthwhile application for investigation in reference to analysing the different effects caused by the two different TLS measurement types.

\section{ACKNOWLEDGEMENTS}

The Wajarri Traditional Land Owners are thanked for allowing us access to Walga Rock for the data collection and for permitting the completion of the project. The Weld Range, Web of Knowledge is also acknowledged for their collaboration and recommendations. In addition, thanks go to the Curtin University 2014 Survey Expedition that made the necessary equipment available and the data collection possible.

\section{REFERENCES}

Armesto-González, J., Riveiro-Rodríguez, B., GonzálezAguilera, D., \& Rivas-Brea, M. T. (2010). Terrestrial laser scanning intensity data applied to damage detection for historical buildings. Journal of Archaeological Science, 37(12), 3037-3047. doi:10.1016/j.jas.2010.06.031

Boehler, W., Vicent, M. B., \& Marbs, A. (2003). Investigating laser scanner accuracy. The International Archives of Photogrammetry, Remote Sensing and Spatial Information Sciences, 34(Part 5), 696-701.

Briese, C., Doneus, M., Pfennigbauer, A., Ullrich, A., \& Doneus, A. (2013, 6/09/2013). Multi-wavelength airborne laser scanning for archaeological prospection. Paper presented at the XXIV International CIPA Symposium, Strasbourg, France.

Burton, D., Dunlap, D. B., Wood, L. J., \& Flaig, P. P. (2011). Lidar Intensity as a Remote Sensor of Rock Properties. Journal of Sedimentary Research, 81(5), 339-347. doi:10.2110/jsr.2011.31

Fang, W., Huang, X., Zhang, F., \& Li, D. (2015). Intensity Correction of Terrestrial Laser Scanning Data by Estimating Laser Transmission Function. Geoscience and Remote Sensing, IEEE Transactions on, 53(2), 942-951. doi:10.1109/TGRS.2014.2330852

Franceschi, M., Teza, G., Preto, N., Pesci, A., Galgaro, A., \& Girardi, S. (2009). Discrimination between marls and limestones using intensity data from terrestrial laser scanner. ISPRS Journal of Photogrammetry and Remote Sensing, 64(6), 522-528. doi:10.1016/j.isprsjprs.2009.03.003

Gerke, M., \& Xiao, J. (2014). Fusion of airborne laserscanning point clouds and images for supervised and unsupervised scene classification. ISPRS Journal of Photogrammetry and Remote Sensing, $\quad 87, \quad 78-92$ doi:http://dx.doi.org/10.1016/j.isprsjprs.2013.10.011

González-Jorge, H., Gonzalez-Aguilera, D., RodriguezGonzalvez, P., \& Arias, P. (2012). Monitoring biological crusts in civil engineering structures using intensity data from terrestrial laser scanners. Construction and Building Materials, 31, 119-128. doi:10.1016/j.conbuildmat.2011.12.053

Hartzell, P., Glennie, C., Biber, K., \& Khan, S. (2014). Application of multispectral LiDAR to automated virtual outcrop geology. ISPRS Journal of Photogrammetry and Remote Sensing, $\quad 88, \quad$ 147-155. doi:10.1016/j.isprsjprs.2013.12.004

Hartzell, P., Glennie, C., \& Finnegan, D. C. (2015). Empirical Waveform Decomposition and Radiometric Calibration of a Terrestrial Full-Waveform Laser Scanner. IEEE Trans. Geosci. Remote Sensing, 53(1), 162-172. doi:10.1109/TGRS.2014.2320134

Höfle, B., \& Pfeifer, N. (2007). Correction of laser scanning intensity data: Data and model-driven approaches. ISPRS Journal of Photogrammetry and Remote Sensing, 62(6), 415433. doi:10.1016/j.isprsjprs.2007.05.008 
Jazayeri, I., \& Fraser, C. S. (2010). Interest operators for feature-based matching in close range photogrammetry. The Photogrammetric Record, 25(129), 24-41.

Kaasalainen, S., Jaakkola, A., Kaasalainen, M., Krooks, A., \& Kukko, A. (2011). Analysis of Incidence Angle and Distance Effects on Terrestrial Laser Scanner Intensity: Search for Correction Methods. Remote Sensing, 3(10), 2207-2221. doi:10.3390/rs3102207

Kaasalainen, S., Niittymaki, H., Krooks, A., Koch, K., Kaartinen, H., Vain, A., \& Hyyppa, H. (2010). Effect of Target Moisture on Laser Scanner Intensity. IEEE Trans. Geosci. Remote Sensing, 48(4), 2128-2136. doi:10.1109/TGRS.2009.2036841

Kukko, A., Kaasalainen, S., \& Litkey, P. (2008). Effect of incidence angle on laser scanner intensity and surface data. Applied optics, 47(7), 986.

Lerma, J. L. (2001). Multiband versus multispectral supervised classification of architectural images. The Photogrammetric Record, 17(97), 89-101.

Lu, D., \& Weng, Q. (2007). A survey of image classification methods and techniques for improving classification performance. International Journal of Remote Sensing, 28(5), 823-870.

Luhmann, T., Robson, S., Kyle, S., \& Boehm, J. (2013). Closerange photogrammetry and $3 D$ imaging: Walter de Gruyter.

MATLAB R2014a (Version 8.4.0.150421). (2014).

Soudarissanane, S., Lindenbergh, R., Menenti, M., \& Teunissen, P. (2011). Scanning geometry: Influencing factor on the quality of terrestrial laser scanning points. ISPRS Journal of Photogrammetry and Remote Sensing, 66(4), 389-399. doi:10.1016/j.isprsjprs.2011.01.005

Teza, G., \& Pesci, A. (2008). Effects of surface irregularities on intensity data from laser scanning: an experimental approach. Annals of Geophysics, 51(5/6), 839-848.

Vosselman, G. V., \& Maas, H.-G. (2010). Airborne and terrestrial laser scanning: Whittles. 